\begin{tabular}{ll} 
C O L L O Q U I U M & M A T H E M A T I C U M \\
\hline vol. LXIX & 1995 \\
\hline
\end{tabular}

\title{
RADIAL GROWTH AND VARIATION OF UNIVALENT FUNCTIONS \\ AND OF DIRICHLET FINITE HOLOMORPHIC FUNCTIONS
}

BY

DANIEL GIRELA (MÁLAGA)

A well known result of Beurling asserts that if $f$ is a function which is analytic in the unit disc $\Delta=\{z \in \mathbb{C}:|z|<1\}$ and if either $f$ is univalent or $f$ has a finite Dirichlet integral then the set of points $e^{i \theta}$ for which the radial variation

$$
V\left(f, e^{i \theta}\right)=\int_{0}^{1}\left|f^{\prime}\left(r e^{i \theta}\right)\right| d r
$$

is infinite is a set of logarithmic capacity zero. In this paper we prove that this result is sharp in a very strong sense. Also, we prove that if $f$ is as above then the set of points $e^{i \theta}$ such that

$$
(1-r)\left|f^{\prime}\left(r e^{i \theta}\right)\right| \neq o(1) \quad \text { as } r \rightarrow 1
$$

is a set of logarithmic capacity zero. In particular, our results give an answer to a question raised by T. H. MacGregor in 1983.

1. Introduction and statement of results. Let $\Delta$ denote the unit $\operatorname{disc}\{z \in \mathbb{C}:|z|<1\}$. Let $\mathcal{D}$ be the family of functions $f$ holomorphic in $\Delta$ with finite Dirichlet integral, that is, those which satisfy

$$
\iint_{\Delta}\left|f^{\prime}(z)\right|^{2} d x d y<\infty
$$

The radial growth of the derivative of a function in $\mathcal{D}$ has been studied by several authors. In [5], [6], [8] and [13] it was proved by different methods that if $f \in \mathcal{D}$ then

$$
\left|f^{\prime}\left(r e^{i \theta}\right)\right|=o\left(\frac{1}{(1-r)^{1 / 2}}\right) \quad \text { as } r \rightarrow 1,
$$

\footnotetext{
1991 Mathematics Subject Classification: 30D40, 30C35.

Key words and phrases: radial variation, Dirichlet integral, univalent functions, ca-

This research has been supported in part by a D.G.I.C.Y.T. grant (PB91-0413) and by a grant from "La Junta de Andalucía."
} pacity. 
for almost every $\theta$. Furthermore, in [7] and [8] it is shown that this result is sharp in a very strong sense.

Seidel and Walsh [13] proved that (1.1) holds for almost every $\theta$ if $f$ is analytic and univalent in $\Delta$. However, this result is not sharp: it was first improved by Clunie and MacGregor [2] and later by Makarov [11] who proved that if $f$ is analytic and univalent in $\Delta$ then

$$
\left|\log f^{\prime}\left(r e^{i \theta}\right)\right|=O\left\{\left(\log \frac{1}{1-r} \log \log \log \frac{1}{1-r}\right)^{1 / 2}\right\} \quad \text { as } r \rightarrow 1,
$$

for almost every $\theta$, and that this result is sharp.

So far our discussion has emphasized exceptional sets of measure zero and the results obtained for univalent functions are quite different from those obtained for functions with finite Dirichlet integral. Now, we shall consider the corresponding problems for other kinds of exceptional sets. Namely, we shall be concerned with sets of logarithmic capacity zero and we shall see that in this setting the results for the space $\mathcal{D}$ will be very close to those for univalent functions. We refer to [12] and [15] for the basic results about logarithmic capacity. For brevity, let $\mathcal{U}$ denote the family of all functions $f$ which are analytic and univalent in $\Delta$.

If $f$ is an analytic function in $\Delta$ and $\theta \in[-\pi, \pi]$, we define

$$
V\left(f, e^{i \theta}\right)=\int_{0}^{1}\left|f^{\prime}\left(r e^{i \theta}\right)\right| d r .
$$

Then $V\left(f, e^{i \theta}\right)$ denotes the radial variation of $f$ on the radius of $\Delta$ which terminates at the point $e^{i \theta}$. The following well known result is due to Beurling [1] (see also [14], Chapter 3 of [3] and Chapter VIII of [15]).

Theorem A. Let $f$ be a function which is analytic in $\Delta$. If $f \in \mathcal{D} \cup \mathcal{U}$, then:

(i) The set of points $e^{i \theta}$ for which $V\left(f, e^{i \theta}\right)=\infty$ is of logarithmic capacity zero.

(ii) The set of points $e^{i \theta}$ such that $f$ fails to have finite non-tangential limit $f\left(e^{i \theta}\right)$ at $e^{i \theta}$ is also of logarithmic capacity zero. Furthermore, if $f$ is univalent, $f\left(e^{i \theta}\right)$ cannot be constant on a set of positive capacity.

Lohwater and Piranian [9] proved the following result which shows that it is not possible to prove anything much stronger than (1.1) valid for any univalent function $f$ off sets of capacity zero.

THEOREM B. There exist a function $f$ analytic and univalent in $\Delta$ and a set $E \subset \partial \Delta$ of positive logarithmic capacity such that

$$
\lim _{r \rightarrow 1}(1-r)^{1 / 2}\left|f^{\prime}\left(r e^{i \theta}\right)\right|=\infty \quad \text { for all } e^{i \theta} \in E .
$$


In view of this result, MacGregor raised the following question [10, p. 75].

Is there a number $\alpha(1 / 2<\alpha<3)$ such that each analytic univalent function $f$ satisfies $(1-r)^{\alpha}\left|f^{\prime}\left(r e^{i \theta}\right)\right|=O(1)(r \rightarrow 1)$, except for some set of values of $\theta$ having capacity zero?

Our first result implies that the answer to this question is affirmative, with $\alpha=1$.

THEOREM 1. Let $f$ be a function which is analytic in $\Delta$. If $f \in \mathcal{D} \cup \mathcal{U}$ then the set of points $e^{i \theta}$ such that

$$
(1-r)\left|f^{\prime}\left(r e^{i \theta}\right)\right| \neq o(1) \quad \text { as } r \rightarrow 1
$$

is of logarithmic capacity zero.

Our next result improves Theorem B and shows that Theorem A(i) and Theorem 1 are sharp in a very strong sense and, in particular, implies that 1 is the greatest possible value of $\alpha$ for which MacGregor's condition is satisfied.

ThEOREM 2. Let $\phi:[0,1) \rightarrow(0, \infty)$ be an increasing function and let $\Psi:[1, \infty) \rightarrow(0, \infty)$ be defined by the relation

$$
\phi(r)=\Psi\left(\frac{1}{1-r}\right), \quad 0 \leq r<1 .
$$

Suppose that

$$
\begin{gathered}
(1-r) \phi(r) \text { is decreasing, } \\
\int_{0}^{1} \phi(r) d r<\infty, \\
\Psi(x)-\log \left(\Psi\left(x e^{\Psi(x)}\right)\right) \rightarrow \infty \quad \text { as } x \rightarrow \infty .
\end{gathered}
$$

Then there exist a function $f$ analytic, univalent and bounded in $\Delta$ (hence $f \in \mathcal{D} \cap \mathcal{U})$ and a closed set $\Gamma \subset \partial \Delta$ with positive logarithmic capacity such that

$$
\left|f^{\prime}\left(r e^{i \theta}\right)\right| \phi(r)^{-1} \rightarrow \infty \quad \text { as } r \rightarrow 1
$$

for every $e^{i \theta} \in \Gamma$.

We remark that, if $n$ is a positive integer and $\beta>1$, then the function $\phi$ of Theorem 1 can be taken to be

$$
\phi(r)=\frac{1}{(1-r)\left(\log \frac{1}{1-r}\right)\left(\log \log \frac{1}{1-r}\right) \ldots(\underbrace{\left.\log \ldots \log \frac{1}{1-r}\right)^{\beta}}_{n \text { times }}}
$$

for all $r$ sufficiently close to 1 . 
2. Proof of the results. First of all, let us fix some notation. If $I$ is an interval of $\mathbb{R}$ then $|I|$ will denote the length (one-dimensional Lebesgue measure) of $I$. Also, if $G$ is any Borel subset of $\mathbb{C}, \operatorname{Cap}(G)$ will denote the (inner) logarithmic capacity of $G$, that is,

$$
\operatorname{Cap}(G)=\sup _{\substack{F \subset E \\ F \text { closed }}} \operatorname{Cap}(F) .
$$

Proof of Theorem 1 . Let $f$ be as in Theorem 1 and let $F_{f}$ be the Fatou set of $f$, that is,

$$
F_{f}=\left\{e^{i \theta}: f \text { has a finite non-tangential limit at } e^{i \theta}\right\} .
$$

A result of Zygmund [16, p. 181] proves that

$$
(1-r)\left|f^{\prime}\left(r e^{i \theta}\right)\right| \rightarrow 0 \quad \text { as } r \rightarrow 1 \text { for all } e^{i \theta} \in F_{f} .
$$

Using Theorem A(ii), we see that $\partial \Delta \backslash F_{f}$ has logarithmic capacity zero. This and (2.1) prove that the set of those $e^{i \theta}$ which satisfy (1.3) has logarithmic capacity zero. This finishes the proof of Theorem 1.

The following lemma is a generalization of the lemma proved in $[9, \mathrm{p}$. $592]$ and will be used in the proof of Theorem 2 .

LEMMA 1. Let $\phi:[0,1) \rightarrow(0, \infty)$ be an increasing function and let $F$ be a $2 \pi$-periodic function defined on $\mathbb{R}$ which is increasing in $(-\pi, \pi]$. Let $u$ be the Poisson-Stieltjes integral $\mathbf{P}(d F)$, that is,

$$
u\left(r e^{i \theta}\right)=\frac{1}{2 \pi} \int_{-\pi}^{\pi} \frac{1-r^{2}}{1+r^{2}-2 r \cos (\theta-t)} d F(t), \quad 0 \leq r<1, \theta \in \mathbb{R} .
$$

Let $\theta_{0} \in(-\pi, \pi)$ and suppose that

$$
\frac{F\left(\theta_{0}+h\right)-F\left(\theta_{0}-h\right)}{h \phi(1-h)} \rightarrow \infty \quad \text { as } h \rightarrow 0^{+} .
$$

Then

$$
u\left(r e^{i \theta_{0}}\right) \phi(r)^{-1} \rightarrow \infty \quad \text { as } r \rightarrow 1 .
$$

Proof. We may assume without loss of generality that $\theta_{0}=0$. Then, for $0<r<1$, we have

$$
u(r)=\frac{1}{2 \pi} \int_{-\pi}^{\pi} \frac{1-r^{2}}{1+r^{2}-2 r \cos t} d F(t) \geq \frac{1}{2 \pi} \int_{-(1-r)}^{1-r} \frac{1-r^{2}}{1+r^{2}-2 r \cos t} d F(t),
$$

which, upon using the simple inequality

$$
\frac{1-r^{2}}{1+r^{2}-2 r \cos t} \geq \frac{1-r^{2}}{(1-r)^{2}+r t^{2}} \geq \frac{1}{1-r}, \quad|t|<1-r,
$$


implies

$$
\begin{aligned}
u(r) & \geq \frac{1}{2 \pi} \int_{-(1-r)}^{1-r} \frac{1}{1-r} d F(t) \\
& =\frac{1}{2 \pi} \frac{1}{1-r}[F(1-r)-F(-(1-r))], \quad 0<r<1 .
\end{aligned}
$$

Consequently, we obtain

$$
u(r) \phi(r)^{-1} \geq \frac{1}{2 \pi} \frac{1}{(1-r) \phi(r)}[F(1-r)-F(-(1-r))], \quad 0<r<1 .
$$

Since $\theta_{0}=0$, by $(2.2)$ this implies $u(r) \phi(r)^{-1} \rightarrow \infty$ as $r \rightarrow 1$.

Proof of Theorem 2. Let $\phi$ and $\Psi$ be as in Theorem 2. It is trivial to see that, in addition to (1.7),

$$
\begin{gathered}
\Psi \text { is increasing in }[1, \infty), \\
\Psi(x) / x \text { is decreasing in }[1, \infty), \\
\int_{1}^{\infty} \frac{\Psi(x)}{x^{2}} d x<\infty .
\end{gathered}
$$

\section{Define}

$$
p_{0}=1, \quad p_{k}=\exp \Psi\left(2^{k}\right), \quad k \geq 1 .
$$

Using (2.4) we see that

$$
\begin{aligned}
\int_{1}^{\infty} \frac{\Psi(x)}{x^{2}} d x & =\sum_{k=0}^{\infty} \int_{2^{k}}^{2^{k+1}} \frac{\Psi(x)}{x^{2}} d x \geq \sum_{k=0}^{\infty} \Psi\left(2^{k}\right) \int_{2^{k}}^{2^{k+1}} \frac{1}{x^{2}} d x \\
& =\frac{1}{2} \sum_{k=0}^{\infty} \frac{\Psi\left(2^{k}\right)}{2^{k}}
\end{aligned}
$$

which, together with (2.6) and (2.7), implies

$$
\sum_{k=0}^{\infty} \frac{\log p_{k}}{2^{k}}<\infty
$$

Now we take a linear Cantor set $E$ constructed as follows. We set $E_{0}=$ $[0,1]$. Now, remove an open interval $J_{1}^{1}$ of length $1-1 / p_{1}$ from $E_{0}$ so that $E_{0} \backslash J_{1}^{1}$ is the union of two closed intervals $I_{1}^{1}, I_{2}^{1}$ of length $1 /\left(2 p_{1}\right)$. We set

$$
E_{1}=I_{1}^{1} \cup I_{2}^{1}=E_{0} \backslash J_{1}^{1} .
$$

Now, for $k=1,2$ we remove an open interval $J_{k}^{2}$ of length $\left|I_{k}^{1}\right|\left(1-1 / p_{2}\right)$ from $I_{k}^{1}$ so that $I_{k}^{1} \backslash J_{k}^{2}$ is the union of two closed intervals $I_{2 k-1}^{2}, I_{2 k}^{2}$ of 
length $\left|I_{k}^{1}\right| /\left(2 p_{2}\right)$. Then we set

$$
E_{2}=\bigcup_{k=1}^{4} I_{k}^{2}=E_{1} \backslash \bigcup_{k=1}^{2} J_{k}^{2} .
$$

We repeat this procedure to obtain an infinite sequence of nested closed sets $E_{0} \supset E_{1} \supset E_{2} \supset \ldots$ At stage $n$ we have

$$
E_{n}=\bigcup_{k=1}^{2^{n}} I_{k}^{n}=E_{n-1} \backslash \bigcup_{k=1}^{2^{n-1}} J_{k}^{n},
$$

where each $I_{k}^{n}$ is a closed interval and each $J_{k}^{n}$ is an open interval, $J_{k}^{n} \subset I_{k}^{n-1}$,

$$
\begin{aligned}
\left|I_{k}^{n}\right| & =\frac{1}{2^{n} p_{1} \ldots p_{n}}, \\
\left|J_{k}^{n}\right| & =\frac{1}{2^{n-1} p_{1} \ldots p_{n-1}}\left(1-\frac{1}{p_{n}}\right) .
\end{aligned}
$$

Finally, we set $E=\bigcap_{n=1}^{\infty} E_{n}$.

Let $F$ be the nondecreasing singular Cantor function associated with the set $E$. Hence

$$
F(t)=\frac{2 k-1}{2^{n}} \text { if } t \in J_{k}^{n}, k=1, \ldots, 2^{n-1}, n=1,2, \ldots
$$

We extend $F$ to $[-\pi, \pi]$ by defining $F(t)=0$ if $-\pi<t \leq 0$ and $F(t)=1$ if $1 \leq t \leq \pi$. We have

LEMma 2. If $t \in E$ then

$$
\frac{F(t+h)-F(t-h)}{h \phi(1-h)} \rightarrow \infty \quad \text { as } h \rightarrow 0^{+} .
$$

Pr o of. Take $t \in E$. For simplicity, suppose that $t \neq 0,1$. Let $h$ be a positive number so small that $0<t-h<t+h<1$. Since $t \in E$, for each $n$ there exists $k_{n} \in\left\{1, \ldots, 2^{n}\right\}$ such that $t \in I_{k_{n}}^{n}$. Let $N=N(h)$ be the natural number such that

$$
t \in I_{k_{N}}^{N} \subset(t-h, t+h),
$$

but

$$
I_{k_{N-1}}^{N-1} \not \subset(t-h, t+h) .
$$

We easily see that (2.15) implies

$$
h \leq\left|I_{k_{N-1}}^{N-1}\right|=\frac{1}{2^{N-1} p_{1} \ldots p_{N-1}} .
$$


On the other hand, (2.14) implies that the interval $I_{k_{N}}^{N}$ separates the points $t-h$ and $t+h$ and then it follows easily that

$$
F(t+h)-F(t-h) \geq \frac{1}{2^{N}} .
$$

Using (1.4), (2.5) and (2.16) we obtain

$$
h \phi(1-h)=h \Psi\left(\frac{1}{h}\right) \leq \frac{\Psi\left(2^{N-1} p_{0} p_{1} \ldots p_{N-1}\right)}{2^{N-1} p_{0} p_{1} \ldots p_{N-1}},
$$

which, together with (2.17), implies

$$
\frac{F(t+h)-F(t-h)}{h \phi(1-h)} \geq \frac{1}{2} \frac{p_{0} p_{1} \ldots p_{N-1}}{\Psi\left(2^{N-1} p_{0} p_{1} \ldots p_{N-1}\right)} .
$$

Since, clearly, $N \rightarrow \infty$ as $h \rightarrow 0$, using (2.18) we see that (2.13) will follow from

$$
\frac{p_{0} p_{1} \ldots p_{n}}{\Psi\left(2^{n} p_{0} p_{1} \ldots p_{n}\right)} \rightarrow \infty \quad \text { as } n \rightarrow \infty
$$

Now, using (2.7), we see that (2.19) is equivalent to

$$
\sum_{k=0}^{n} \Psi\left(2^{k}\right)-\log \Psi\left(2^{n} \exp \left(\sum_{k=0}^{n} \Psi\left(2^{k}\right)\right)\right) \rightarrow \infty \quad \text { as } n \rightarrow \infty .
$$

Hence we proceed to prove (2.20). For simplicity, define $\varphi:(0, \infty) \rightarrow$ $(0, \infty)$ by

$$
\varphi(x)= \begin{cases}\Psi(1) & \text { if } 0<x<1, \\ \Psi(x) / x & \text { if } x \geq 1 .\end{cases}
$$

Then $\varphi$ is decreasing in $(0, \infty)$ and, hence, we have

$$
\begin{aligned}
\int_{0}^{2^{n+1}} \varphi(x) d x & =\varphi(1)+\sum_{k=0}^{n} \int_{2^{k}}^{2^{k+1}} \varphi(x) d x \\
& \leq \varphi(1)+\sum_{k=0}^{n} \varphi\left(2^{k}\right) 2^{k}=\varphi(1)+\sum_{k=0}^{n} \Psi\left(2^{k}\right) .
\end{aligned}
$$

Since $\varphi$ is decreasing, we easily obtain

$$
\Psi(s) \leq \int_{0}^{s} \varphi(x) d x, \quad s \geq 1 .
$$

Using (2.22) and (2.23), we see that

$$
2^{n} \exp \Psi\left(2^{n+1}\right) \leq 2^{n} e^{\varphi(1)} \exp \left(\sum_{k=0}^{n} \Psi\left(2^{k}\right)\right)
$$


which, upon using (2.5), implies

$$
\frac{\Psi\left(2^{n} \exp \left[\Psi\left(2^{n+1}\right)\right]\right)}{2^{n} \exp \left[\Psi\left(2^{n+1}\right)\right]} \geq \frac{\Psi\left(2^{n} e^{\varphi(1)} \exp \left[\sum_{k=0}^{n} \Psi\left(2^{k}\right)\right]\right)}{2^{n} e^{\varphi(1)} \exp \left[\sum_{k=0}^{n} \Psi\left(2^{k}\right)\right]},
$$

or, equivalently,

$$
\begin{aligned}
\log \Psi\left(2^{n} \exp \left[\Psi\left(2^{n+1}\right)\right]\right)+\varphi(1)+\sum_{k=0}^{n} \Psi\left(2^{k}\right)-\Psi\left(2^{n+1}\right) & \\
& \geq \log \Psi\left(2^{n} e^{\varphi(1)} \exp \left[\sum_{k=0}^{n} \Psi\left(2^{k}\right)\right]\right) .
\end{aligned}
$$

Since $\Psi$ is increasing, this implies

$$
\begin{aligned}
& \sum_{k=0}^{n} \Psi\left(2^{k}\right)-\log \Psi\left(2^{n} \exp \left[\sum_{k=0}^{n} \Psi\left(2^{k}\right)\right]\right) \\
& \geq \sum_{k=0}^{n} \Psi\left(2^{k}\right)-\log \Psi\left(2^{n} e^{\varphi(1)} \exp \left[\sum_{k=0}^{n} \Psi\left(2^{k}\right)\right]\right) \\
& \geq \Psi\left(2^{n+1}\right)-\log \Psi\left(2^{n} \exp \left[\Psi\left(2^{n+1}\right)\right]\right)-\varphi(1) \\
& \geq \Psi\left(2^{n+1}\right)-\log \Psi\left(2^{n+1} \exp \left[\Psi\left(2^{n+1}\right)\right]\right)-\varphi(1) .
\end{aligned}
$$

Then (2.20) follows from (1.7).

A minor modification of the above argument shows that (2.13) also holds for $t=0$ and for $t=1$. Hence Lemma 2 is proved.

Having established Lemma 2 we continue with the proof of Theorem 2 as follows. Let

$$
\Gamma_{1}=\left\{e^{i t}: t \in E\right\} .
$$

Then $\Gamma_{1}$ is a closed subset of $\partial \Delta$. Notice that the set $E$ is $E\left(p_{0} p_{1} \ldots\right)$ in the notation of Chapter V of [12] and hence, using Theorem 3 on p. 153 of [12], we see that (2.8) implies that

$$
\operatorname{Cap}\left(\Gamma_{1}\right)>0 .
$$

We set $u=\mathbf{P}(d F)$ and we let $g$ be the function which is analytic in $\Delta$ with $g(0) \in \mathbb{R}$ and $\Re g=u$. Set

$$
h(z)=\int_{[0, z]} g(\xi) d \xi, \quad z \in \Delta .
$$

Since $\Re h^{\prime}=u>0$, it is clear that $h$ is univalent in $\Delta$ and using Lemmas 2 and 1 , we deduce that

$$
\left|h^{\prime}\left(r e^{i \theta}\right)\right| \phi(r)^{-1} \rightarrow \infty \quad \text { as } r \rightarrow 1, \text { for every } e^{i \theta} \in \Gamma_{1} .
$$


To finish the proof of Theorem 2 we use a standard method which relates any univalent function to a bounded univalent function (see e.g. [4, p. 11] or $\left[10\right.$, p. 70]). Let $c \in \mathbb{C} \backslash h(\Delta)$. Then $h_{1}=(h-c)^{1 / 2}$ is analytic and univalent in $\Delta$. Moreover, $h_{1}$ has the property that if $\xi \in h_{1}(\Delta)$ then $-\xi \notin h_{1}(\Delta)$ and then, since $h_{1}$ is an open mapping, we deduce that there exists $d \in \mathbb{C}$ and $r>0$ such that $h_{1}(\Delta)$ does not meet the disc $\{w \in \mathbb{C}:|w-d|<r\}$. Consequently, the function $f=1 /\left(h_{1}-d\right)$ is analytic, univalent and bounded in $\Delta$ and we have

$$
\left|f^{\prime}\right|=\frac{1}{2}|h-c|^{-1 / 2}\left|h_{1}-d\right|^{-2}\left|h^{\prime}\right| .
$$

Let $G_{1}$ be the set of those $e^{i \theta}$ such that $h$ has a finite non-tangential limit $h\left(e^{i \theta}\right)$ not equal to $c$, let $G_{2}$ be the set of those $e^{i \theta}$ such that $h_{1}$ has a finite non-tangential limit $h\left(e^{i \theta}\right)$ not equal to $d$ and let $F_{j}=\partial \Delta \backslash G_{j}(j=1,2)$. Clearly, (2.26) and (2.27) show that

(2.28) $\left|f^{\prime}\left(r e^{i \theta}\right)\right| \phi(r)^{-1} \rightarrow \infty \quad$ as $r \rightarrow 1$, for every $e^{i \theta} \in \Gamma_{1} \backslash\left(F_{1} \cup F_{2}\right)$.

Having in mind that $h$ and $h_{1}$ are univalent and using Theorem A(ii), we see that $F_{1}$ and $F_{2}$ are sets of logarithmic capacity zero and hence

$$
\operatorname{Cap}\left(\Gamma_{1} \backslash\left(F_{1} \cup F_{2}\right)\right)>0 .
$$

Now take a closed set $\Gamma \subset \Gamma_{1} \backslash\left(F_{1} \cup F_{2}\right)$ with $\operatorname{Cap}(\Gamma)>0$. Clearly (2.28) implies

$$
\left|f^{\prime}\left(r e^{i \theta}\right)\right| \phi(r)^{-1} \rightarrow \infty \quad \text { as } r \rightarrow 1, \text { for every } e^{i \theta} \in \Gamma .
$$

This finishes the proof.

\section{REFERENCES}

[1] A. Beurling, Ensembles exceptionnels, Acta Math. 72 (1940), 1-13.

[2] J. Clunie and T. H. MacGregor, Radial growth of the derivative of univalent functions, Comment. Math. Helv. 59 (1984), 362-375.

[3] E. F. Collingwood and A. J. Lohwater, The Theory of Cluster Sets, Cambridge University Press, London, 1966.

[4] P. L. Duren, Univalent Functions, Springer, New York, 1983.

[5] T. M. Flett, On the radial order of a univalent function, J. Math. Soc. Japan 11 (1959), 1-3.

[6] F. W. Gehring, On the radial order of subharmonic functions, ibid. 9 (1957), 77-79.

[7] D. Girela, On analytic functions with finite Dirichlet integral, Complex Variables Theory Appl. 12 (1989), 9-15.

[8] D. J. Hallen beck and K. Samotij, Radial growth and variation of Dirichlet finite holomorphic functions in the disk, Colloq. Math. 58 (1990), 317-325.

[9] A. J. Lohwater and G. Piranian, On the derivative of a univalent function, Proc. Amer. Math. Soc. 4 (1953), 591-594. 
[10] T. H. MacGregor, Radial growth of a univalent function and its derivatives off sets of measure zero, in: Contemp. Math. 38, Amer. Math. Soc., 1985, 69-76.

[11] N. G. Makarov, On the distortion of boundary sets under conformal mappings, Proc. London Math. Soc. (3) 51 (1986), 369-384.

[12] R. Nevanlinna, Analytic Functions, Springer, New York, 1970.

[13] W. Seidel and J. L. Walsh, On the derivatives of functions analytic in the unit disc and their radii of univalence and of p-valence, Trans. Amer. Math. Soc. 52 (1942), 128-216.

[14] M. Tsuji, Beurling's theorem on exceptional sets, Tôhoku Math. J. 2 (1950), 113125.

[15] —, Potential Theory in Modern Function Theory, Chelsea, New York, 1975.

[16] A. Zygmund, On certain integrals, Trans. Amer. Math. Soc. 55 (1944), 170-204.

ANÁLISIS MATEMÁTICO

FACULTAD DE CIENCIAS

UNIVERSIDAD DE MÁLAGA

29071 MÁLAGA, SPAIN 